\title{
RELACIÓN DE LOS MOROS Y CRISTIANOS SUJETOS AL IMPUESTO DEL MORABATÍ EN «LO LOCH DE ONDARA» EN 1404
}

Por

MARIAA JESÚS PATERNINA BONO

El presente documento nos ha sido facilitado por don Roberto Miralles i Cebrià, historiador y actual Alcalde de Ondara. Se trata de la relación de los habitantes de dicho lugar sujetos al impuesto del morabatí en 1404 que se conserva en el Archivo del Reino de Valencia (1).

Por considerar de interés el listado a varios niveles: onomástico, económico, demográfico, etc. es por lo que nos decidimos a su publicación con el único propósito de aportar nuevas fuentes a los diversos estudiosos que actualmente están trabajando en este interesante campo de la onomástica, y de la árabe en particular.

Dicha relación ocupa un total de nueve folios en los que aparecen los nombres de los cristianos primero, seguidos de los moros, que pagaron el tributo.

El documento está estructurado en dos columnas, a la izquierda los nombres y apellidos del impositor y a la derecha la cantidad (igual para todos) de un morabatí (2); al final de cada folio hay una nota con la suma de las cantidades parciales. En el último folio, y después de la cantidad correspondiente, aparece la suma total de la relación. Aunque las sumas parciales son correctas, en la final existe una diferencia de un morabatí de menos.

(1) Archivo del Reino de Valencia. Maestre Racional. Exped. n. ${ }^{\circ} 9,600$, ff. 177-181.

(2) Para el estudio del impuesto del morabatí ver, entre otros, los trabajos de López Elum y Mateu Llopis citados en la bibliografía. 
Cierra el documento Ramón Vidal, notario que hizo la relación, clausurando la misma con su signo.

Nuestro trabajo ha consistido en la transcripción de la lista y en confeccionar una relación alfabética de todos los nombres y apellidos - tanto cristianos como moros - que aparecen en la misma, así como su frecuencia, numerándolos para facilitar su localización.

Es frecuente en esta relación que la manera de identificar a las personas que han pagado el tributo sea por su relación de parentesco bien con el sujeto inmediato anterior (sa sogra, son fill, sa mare) bien por ser "la muller de....; nos ha parecido oportuno por tanto el confeccionar otra lista con estas relaciones familiares. La profesión sólo se indica en dos ocasiones: sastre (C-25) $y$ "lo texidor" (C-26), y en un caso aparece el cargo: batle (c-23).

Hemos comparado los antropónimos árabes de esta relación con los listados del documentado y exhaustivo trabajo de Ana Labarta (3) y vemos que la mayoría de ellos no aparecen en el mismo o están escritos con grafías distintas Abrafim LAbrahim, Abraxim?; esto último nos plantea el problema de la transcripción fonética de la onomástica árabe por un escriba de lengua valenciana [ver para los problemas filológicos la tesis doctoral de Carmen Barceló (4)].

Otra de las dificultades para la identificación de los nombres árabes ha sido el tenerles que aplicar para su desarrollo, en los casos de aparecer abreviados, las normas de las abreviaturas latinas.

La relación de Ondara está compuesta por un total de 120 impositores que se dividen en 30 cristianos y 90 moros. Hemos relacionado 31 nombres distintos entre los cristianos y 88 entre los moros; en cuanto a su frecuencia se puede establecer el siguiente orden:

Cristianos. Nombres: Pere, Jacme, Gonçalo, Johan, Ramón y Ramona. Apellidos: Pelegrí, Martí y Vidal.

Moros. Nombres: Caat, Mahomat, Ali, Azmet, Coot.

Apellidos: Alcater, Yaye, Xadit.

Para terminar quiero dar mis más expresivas gracias al profesor Míkel de Epalza, sin cuyo estímulo, consejo y asesoramiento este pequeño trabajo no hubiera sido posible.

(3) LABARTA, Ana: La onomástica de los moriscos valencianos. Madrid, 1987.

(4) BARCELÓ TORRES, M. ${ }^{8}$ del Carmen: Minorias islámicas en el País Valenciano: Historia y dialecto. Valencia, 1984. 


\section{Lo loch de Ondara}

\section{LOS CHRISTIANS}

\begin{tabular}{|c|c|c|}
\hline $\mathrm{C}-1$ & Pere Pelegrí ........... & 1 morabatí \\
\hline $\mathrm{C}-2$ & 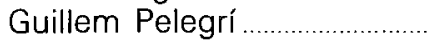 & 1 morabatí \\
\hline $\mathrm{C}-3$ & La muller d'en Pere Steve & 1 morabatí \\
\hline C-4 & $\mathrm{Na}$ Ramona & 1 morabatí \\
\hline-5 & Son fill.............. & 1 mora \\
\hline C-6 & La neta de na Ramona & \\
\hline C-7 & Jacme Ferrada.. & \\
\hline C-8 & Roger Ortoneda & \\
\hline $\begin{array}{ll}C-9 \\
C-10\end{array}$ & La dona que te en sa casa & \\
\hline
\end{tabular}

Suma de plana $X$ morabatins // 177

C-11 Gonçalo Pastor ................................................................... 1 morabatí

C-12 Arnau Gavila ................................................................................ 1 morabatí

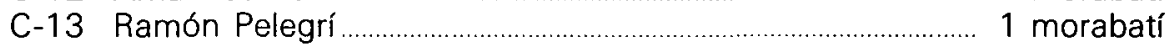

C-14 Francesch Mir ............................................................................... 1 morabatí

C-15 Johan Gençor ............................................................................ 1 morabatí

C-16 Na Jorneta

C-17 Son fill .................................................................................................. 1 morabatí

C-18 Johan García .................................................................... 1 morabatí

C-19 Sa sobra

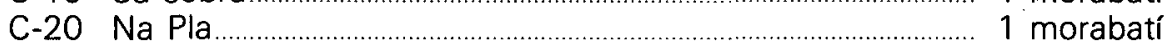

C-21 La muller d'en Jacme Mulet .................................................. 1 morabatí

C-22 Gonçalo Gavila ............................................................................ 1 morabatí

C-23 Ramón Vidal, batle...................................................................... 1 morabatí

Suma de plana XIII morabatins // 177v.

C-24 Ferrando Peric ............................................................................. 1 morabatí

C-25 Bernat Vidal, sastre …......................................................... 1 morabatí

C-26 Nortola, lo texidor .......................................................................... 1 morabatí

C-27 Jacme Roic ....................................................................... 1 morabatí

C-28 Son fill ........................................................................................ 1 morabatí

C-29 Pere Martí .................................................................................... 1 morabatí

C-30 Pere Martí, son fill ..................................................................... 1 morabatí

\section{LOS MOROS}

M-1 Cayt Hayr .................................................................................... 1 morabatí

M-2 Gebelli................................................................................. 1 morabatí

M-3 Sa filla ............................................................................. 1 morabatí

M-4 Zohayr .................................................................................. 1 morabatí

M-5 Ali Allir ............................................................................... 1 morabatí

M-6 Cayt Alacir .................................................................................... 1 morabatí 
Suma de plana XIII morabatins // 178

\begin{tabular}{|c|c|c|}
\hline M-7 & Coaytal Bazqrin.... & 1 morabatí \\
\hline M-8 & Aloçayla ..................... & 1 morabatí \\
\hline M-9 & Son fill to chich. & 1 morabati \\
\hline$M-10$ & Ali Albocayrini...... & 1 morabat \\
\hline$M-11$ & Son fill hereu de la altra muller..... & 1 morabati \\
\hline M-12 & Yacix Zaydon & 1 morabat \\
\hline$M-13$ & Mahomat Xadit ........ & 1 morabat \\
\hline M-14 & 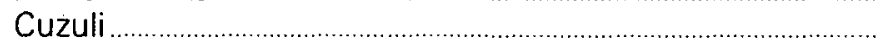 & 1 morabat \\
\hline M-15 & Ayot Albayre. & 1 morabat \\
\hline$M-16$ & Coot Cacin ...... & 1 morabat \\
\hline M-17 & Caat Algagui .......... & 1 morabat \\
\hline M-18 & Mahomat Morabit... & 1 morabat \\
\hline$M-19$ & Mahomat Jeyeni ...... & 1 morabat \\
\hline M-20 & Coot Zaydon.......... & 1 morabat \\
\hline
\end{tabular}

Suma de plana XIIII morabatins /I 178v.

M-21 Son nebot ............................................................................... 1 morabatí

M-22 Caat Alget ............................................................................... 1 morabatí

M-23 Sa mare...................................................................................... 1 morabatí

M-24 Yaye Ananyar ................................................................ 1 morabatí

M-25 Compareta ...................................................................... 1 morabatí

M-26 Mahomat Ell............................................................................. 1 morabatí

M-27 La muller de Bolaix ........................................................... 1 morabatí

M-28 Caat Bolaix .............................................................................. 1 morabatí

M-29 Axer Xadit .................................................................... 1 morabatí

M-30 Juceff Zich Zich ....................................................................... 1 morabatí

M-31 Mahomat Alcater ............................................................ 1 morabatí

M-32 Maymo Cacin ............................................................................ 1 morabatí

M-33 Ayet Abolaix ................................................................. 1 morabatí

M-34 Caat Bachtoza .......................................................................... 1 morabatí

M-35 Azimet Bachtoza ..................................................................... 1 morabatí

Suma de plana XV morabatins // 179

M-36 Catal

1 morabatí

M-37 Alguagui

1 morabatí

M-38 La muller de Abzequendel ..................................................

M-39 Caat Albarber ……………………………………………………..... 1 morabatí

M-40 Ubequer Arraic ...................................................................... 1 morabatí

M-41 La muller de Hajuba ............................................................. 1 morabatí

M-42 Cale Abençabe.......................................................................... 1 morabatí

M-43 Sos fills hereus de l'altra muller ............................................... 1 morabatí

M-44 Ali Mochta ........................................................................ 1 morabatí

M-45 Facen son jendre de cacha ..................................................... 1 morabatí

M-46 Azmet Malich .......................................................................... 1 morabatí

M-47 Son nebot ........................................................................... 1 morabatí

M-48 Fanidell Taer ..................................................................................... 1 morabatí 
M-49 Ayen Abenyaren

1 morabatí

M-50 Yaye Alcater

1 morabatí

Suma de plana XV morabatins // 179v.

M-51 Abrafim Zaydo

1 morabatí

M-52 Ali Raro

1 morabatí

M-53 Hayzopara

1 morabatí

M-54 Axer Xadit

1 morabatí

M-55 Yaye Taix

1 morabatí

M-56 Ali Cucen

1 morabatí

M-57 Abdalla Hatap

1 morabatí

M-58 Azmet Rafe

1 morabatí

M-59 Coot Rafe

1 morabatí

M-60 La muller de Nadir

1 morabatí

M-61 Muça

1 morabatí

M-62 Abenjama

1 morabatí

M-63 Caat Morit

1 morabatí

M-64 Calim Xoaya

1 morabatí

M-65 La filla hereua de sa mare

1 morabatí

M-66 Coayat Alcater.

1 morabatí

Suma de plana XVI morabatins // 180

M-67 Ali Albarber

1 morabatí

M-68 Abrafim Alcater ........................................................................... 1 morabatí

M-69 Catan................................................................................... 1 morabatí

M-70 Alaçet .................................................................................. 1 morabatí

M-71 Calim Duqueyech ............................................. 1 morabatí

M-72 Caat Xoaya .................................................................................. 1 morabatí

M-73 Yaye Fudeyl ................................................................... 1 morabatí

M-74 Mahomat Algagui ....................................................................... 1 morabatí

M-75 Coot Dulegi ............................................................................... 1 morabati

M-76 Mahomat Çale ............................................................................... 1 morabatí

M-77 Xagha ........................................................................................ 1 morabatí

M-78 Racbayda .................................................................................... 1 morabati

M-79 Pozoc ….............................................................................................. 1 morabatí

M-80 Azmet Bagua ......................................................................... 1 morabatí

M-81 Meguech ...................................................................................... 1 morabatí

M-82 Cayt Calanta ............................................................................... 1 morabatí

Suma de plana XVI morabatins // 180v.

M-83 Cayt Azeyn

1 morabatí

M-84 Coot Fuley.

1 morabatí

M-85 Caat Fuster

1 morabatí

M-86 Azmet Fuster

1 morabatí

M-87 Azmet Çamaya

1 morabatí

M-88 Axer Nocoron

1 morabatí

M-89 Caat Nadir.

1 morabatí

M-90 Coot Alcater

1 morabatí 
Suma de plana VIII morabatins

Suma de sumes CXVIIII morabatins.

E yo dessus dit Ramon Vidal qui la dessus dita scripcio he feyta, en testimoni de veritat aci he aposat mon signe de notari acostumat.

Sig(signo)num

\section{Al margen}

... a la summa

Índice onomástico

CRISTIANOS

Arnau: C-12

Bernat: C-25

Domingo: $\mathrm{C}-10$

Febrer: C-10

Ferrada: C-7

Ferrando: C-24

Francesch: C-14

García: C-18

Gavila: C-12, C-22

Gençor: C-15

Gonçalo: C-11, C-22

Guillem: C-2

Jacme: C-7, C-21, C-27

Johan: C-15, C-18

Jorneta: C-16

Martí: C-29, C-30

Mir: C-14

Mulet: C-21

Nortola: C-26

Ortoneda: $\mathrm{C}-8$

Pastor: C-11

Pelegrí: C-1, C-2, C-13

Pere: C-1, C-3, C-29, C-30

Peric: C-24

Pla: C-20

Ramón: C-13, C-23

Ramona: C-4, C-6

Roger: $\mathrm{C}-8$

Roic: $\mathrm{C}-27$

Steve: C-3

Vidal: C-23, C-25 
MOROS

Abdalla: $\mathrm{M}-57$

Abençabe: $\mathrm{M}-42$

Abenjama: $M-62$

Abenyaren: $M-49$

Abolaix: $\mathrm{M}-33$

Abrafim: $M-51, M-68$

Abzequendel: $M-38$

Alaçet: $M-70$

Alacir: $M-6$

Albarber: $M-39, M-67$

Albayre: $\mathrm{M}-15$

Albocayrini: $M-10$

Alcater: M-31, M-50, M-66, M-68, M-90

Algagui: $M-17, M-74$

Alget: $M-22$

Alguagui: $M-37$

Ali: M-5, M-10, M-44, M-52, M-56, M-67

Allir: $\mathrm{M}-5$

Aloçayla: $M-8$

Ananyar: $M-24$

Arraic: $M-40$

Axer: $\mathrm{M}-29, \mathrm{M}-54, \mathrm{M}-88$

Ayen: $M-49$

Ayet: $M-33$

Ayot: $\mathrm{M}-15$

Azeyn: $\mathrm{M}-83$

Azimet: $\mathrm{M}-35$

Azmet: M-46, M-58, M-80, M-86, M-87

Bachtoza: M-34, M-35

Bagua: $M-80$

Bazqrin: $M-7$

Bolaix: $M-27, M-28$

Caat: $M-17, M-22, M-26, M-34, M-39, M-63, M-72, M-85, M-89$

Cacim: $M-16, M-32$

Calanta: $\mathrm{M}-82$

Cale: $\mathrm{M}-42$

Cale: $M-76$

Calim: M-64, M-71

Camaya: $\mathrm{M}-87$

Catal: $M-36$

Catan: $M-69$

Cayt: M-1, M-6, M-82, M-83

Coaytal: M-7, M-66

Compareta: $\mathrm{M}-25$

Coot: M-16, M-20, M-59, M-75, M-84, M-90

Cucen: $M-46, M-56$ 
Cuzuli: M-14

Dulegi: M-75

Duqueyech: M-71

Ell: $M-26$

Facen: $\mathrm{M}-45$

Fanidell: $M-48$

Fudeyl: $M-73$

Fuley: M-84

Fuster: M-85, M-86

Gebelli: $M-2$

Hajuba: M-41

Hatap: M-57

Hayr: M-1

Hayzopara: $M-53$

Jeyeni: $M-19$

Juceff: $M-30$

Mahomat: $M-13, M-18, M-19, M-26, M-31, M-74, M-76$

Malich: M-46

Maymo: M-32

Meguech: M-81

Mochta: M-44

Morabit: $\mathrm{M}-18$

Morit: M-63

Muça: $M-61$

Nadir: M-60, M-89

Nocoron: $M-88$

Pozoç: M-79

Racbayda: $M-78$

Rafe: M-58, M-59

Raro: $M-52$

Taer: $M-48$

Taix: M-55

Ubequer: $M-40$

Xadit: M-13, M-29, M-54

Xagha: $M-77$

Xoaya: M-64, M-72

Yacix: $M-12$

Yaye: $M-24, M-50, M-55, M-73$

Zaydo: $M-51$

Zaydon: $M-12, M-20$

Zich Zich: M-30

Zohayr: $M-4$

Relación de parentesco

Dona: $\mathrm{C}-9$

Fill: C-5, C-17, C-28, C-30, M-9, M-11

Filla: M-3, M-65 
Fills: $M-43$

Jendre: $M-45$

Mare: $\mathrm{M}-23$

Muller: C-3, C-21, M-27, M-38, M-41, M-60

Nebot: $M-21, M-47$

Neta: C-6

Sogra: C-19

\section{BIBLIOGRAFÍA}

BARCELO TORRES, M. ${ }^{a}$ del Carmen. Minorias islámicas en el País Valenciano: Historia y dialecto. Valencia, 1984.

GABRIELI, Giuseppe. II nome proprio arabo-musulmano. Roma, 1915.

LABARTA, Ana. La onomástica de los moriscos valencianos. Madrid, 1987.

LÓPEZ ELUM, Pedro. El impuesto del morabati, su base económica y sus aplicaciones demográficas. Datos para su estudio (siglos XIII-XVIII). Valencia, 1972.

MARÍN, Manuela. Volumen colectivo dirigido por... Estudios onomástico-bibliograficos de Al-Andalus. Madrid, 1988.

- "Onomástica árabe en Al-Andalus: Ism 'alam y kunya», Al-Oanțara, Madrid, IV, 1983, 131-149 y "Notas sobre onomástica y denominaciones femeninas en Al-Andalus (siglos VIII-XI)", Homenaje al prof. Darfo Cabanelas..., Granada, 1987, I, 37-52.

MATEU LLOPIS, Felipe. "Morabatí y mazmodina censuales (en especial en el Reino de Valencia)", Cuadernos de la Alhambra, Granada, 10-11, 1974-75, p. 201-215. 